\title{
Electron Holography Study of the Charging Effect in Microfibrils of Sciatic Nerve Tissues
}

\author{
Ki Hyun Kim, ${ }^{1}$ Zentaro Akase, ${ }^{1}$ Daisuke Shindo, ${ }^{1, \star}$ Nobuhiko Ohno, ${ }^{2}$ Yasuhisa Fujii, ${ }^{2}$ \\ Nobuo Terada, ${ }^{2}$ and Shinichi Ohno ${ }^{2}$ \\ ${ }^{1}$ Institute of Multidisciplinary Research for Advanced Materials, Tohoku University, Sendai 980-8577, Japan \\ ${ }^{2}$ Department of Anatomy and Molecular Histology, Interdisciplinary Graduate School of Medicine and Engineering, \\ University of Yamanashi, Chuo, Yamanashi 409-3898, Japan
}

\begin{abstract}
The charging effects of microfibrils of sciatic nerve tissues due to electron irradiation are investigated using electron holography. The phenomenon that the charging effects are enhanced with an increase of electron intensity is visualized through direct observations of the electric potential distribution around the specimen. The electric potential at the surface of the specimen could be quantitatively evaluated by simulation, which takes into account the reference wave modulation due to the long-range electric field.
\end{abstract}

Key words: electron holography, secondary electrons, electric potential distribution, charging effect, microfibril, phase reconstruction, amplitude reconstruction

\section{INTRODUCTION}

The charging effect due to electron irradiation of nonconductive materials such as polymers, ceramics, and biological specimens gives rise to one of the most important problems of electron microscopic observation. The charging effect is generally caused by electron bombardment and the related secondary electron emission. Thus, it is well known that nonconductive thin specimens tend to become positively charged (Kotera et al., 1999; Meyza et al., 2003). This charging effect affects various types of electron microscopy and their related analytical techniques. It is well known that the charging phenomenon, which results in problems such as instability and drift of specimens, hinders the acquisition of reliable analysis data by electron microscopy (Munger, 1977). Therefore, to perform a reliable analysis of charged specimens, in addition to suppressing the charging effect through the use of a metallic coating, evaluation of the magnitude of the charging effect is also important (Wepf et al., 1991; Rodnyansky et al., 2000; Li \& Gu, 2002; Shi et al., 2003; Touzin et al., 2006). Detailed mechanisms for the charging effect in nonconductive materials have not yet been clarified. On the other hand, electron holography provides a unique method to detect the phase shift of electron waves due to electromagnetic fields (Shindo \& Oikawa, 2002). These fields can be recorded on an electron hologram, and then through a reconstruction process the electromagnetic fields within/around a specimen can be analyzed. Therefore, electron holography is expected to be applied for the evaluation of the charging effect in nonconductive materials.

In this study, the charging effect of microfibrils of sciatic nerve tissues with changes in the incident electron

\footnotetext{
() MICROSCOPY SOCIETY OF AMERICA 2013

${ }^{\star}$ Corresponding author. E-mail: shindo@tagen.tohoku.ac.jp

Primary institution where the research was performed is the Institute of Multidisciplinary Research for Advanced Materials, Tohoku University, Japan
}

intensity and morphology of the microfibrils is analyzed by electron holography and bright-field imaging. The electric potential around the specimen with different electron intensity is quantitatively evaluated through electron holographic simulation.

\section{Materials and Methods}

Sciatic nerve tissue specimens of adult C57BL/6 mice were prepared by a quick-freezing method (Ohno et al., 1992). The specimens were freeze-dried (Eiko FD-3AS) overnight at $-95^{\circ} \mathrm{C}$ and under a high vacuum of $10^{-7}$ torr. The freeze-dried specimens were packed in a glass container with an open $\mathrm{OsO}_{4}$ vial (fixation with $\mathrm{OsO}_{4}$ vapor) and incubated for $2 \mathrm{~h}$. The specimens were then sputtered with carbon for $20 \mathrm{~s}$. The sciatic nerve tissue was fixed with carbon tape to a copper plate specimen holder (Shindo et al., 2007). Bright-field imaging and electron holography were carried out using a transmission electron microscope (TEM; JEOL JEM-3000F) with an accelerating voltage of $300 \mathrm{kV}$. The TEM was equipped with a biprism and a Lorentz lens system (Shindo et al., 2003). The spacing between the interference fringes was $\sim 2.4 \mathrm{~nm}$ for a biprism voltage of $50 \mathrm{~V}$, and the spatial resolution and width of the field of view were 7.2 and $460 \mathrm{~nm}$, respectively. The exposure time was set to be constant at $6 \mathrm{~s}$. To obtain the electric potential around the specimens due to electron irradiation, simulations that take into account the reference wave modulation due to the long-range electric field were performed using a mathematical software system (Mathematica, ver. 5.0.1.0, Wolfram Research Inc.).

\section{Results AND Discussion}

Figure 1 shows bright-field images obtained from the microfibril of sciatic nerve tissues with different incident electron intensity. These images were acquired using overfocused 




Figure 1. Bright-field images showing the charging effect on a microfibril of the sciatic nerve tissues with incident electron intensities of (a) 60, (b) 180, (d) 420, and (e) $540 \mathrm{~nm}^{-2} \cdot \mathrm{s}^{-1}$. The insets in $(\mathbf{a}-\mathbf{d})$ show the deflection of the incident electron beam due to the positive charge.

conditions. An increase in the incident electron intensity resulted in a change in the contrast at the edge of the microfibril; in Figure 1b, the bright contrast (indicated by a white arrow) appears at the edge of the microfibril, and in Figure 1c this changes to a dark contrast (indicated by a black arrow) and becomes larger, as shown in Figure 1d. These results indicate that the incident electron beams are more strongly deflected by positive charges that are enhanced by increasing the incident electron intensity. Therefore, it is reasonable to consider that the bright and dark contrasts are caused by deflection of the incident electron beam owing to positive charges that are concentrated at the edge of the microfibril. This tendency is stronger when the specimen is more strongly charged, as shown by the schematic illustrations in the insets of Figures 1a-1d.

Figure 2 presents the electron holography results, which show the electric potential distribution around the microfibril. Electron holograms and the corresponding reconstructed phase images are shown in Figures $2 a-2 c$ and $2 \mathrm{~d}-2 \mathrm{f}$, respectively. Some dark contrasts at the edge of the microfibril are indicated by black arrowheads in the electron holograms. It is considered that these contrasts are caused by the effect similar to that shown in Figure 1. In the reconstructed phase image, the white and black lines denote the equiphase lines formed by electrons passing through the electromagnetic field; in the case of the electric field alone, these lines indicate the equipotential lines reflecting the electric potential distribution integrated along the trajectories of the incident electrons (Shindo \& Oikawa, 2002). Figures $2 \mathrm{~d}-2 \mathrm{f}$ show the electric potential distributions due to the charging effect; these distributions are basically symmetrical around the microfibril. As the incident electron intensity increases, the number of equipotential lines gradually increases, whereas the distance between the equipotential lines decreases. These results indicate that the strength of the electric field increases around the microfibril, which is positively charged owing to electron irradiation. There is some irregular contrast that corresponds to the fluctuation of electric potential in the right top edge as indicated by the arrows in Figures 2e and 2f. This will be pointed out later from the analysis of the amplitude reconstruction process.

Simulations were conducted using a one-dimensional line charge model to quantitatively evaluate the charging effect for the case shown in Figure 2 (Matteucci et al., 1992, 1997; Jackson, 1998). In this system, the reference wave modulation due to the long-range electric field should be considered when the electric potential distribution projected along the incident beam is simulated: $I_{\mathrm{ph}}(\boldsymbol{r})=$ $\cos \left(\phi_{\mathrm{p}}(\boldsymbol{r})-\phi_{\mathrm{p}}(\boldsymbol{r}+\boldsymbol{D})\right)$, where $\phi_{\mathrm{p}}(\boldsymbol{r})$ is the phase change in the incident electron due to electric potential $\varphi_{\mathrm{p}}(\boldsymbol{r})$ and $\phi_{\mathrm{p}}(\boldsymbol{r}+\boldsymbol{D})$ is the phase change in the reference wave modulated by the long-range electric field. $D$ is a vector representing the width of the fringe overlap (Okada et al., 2007). Figure 3 shows the simulation results for electric potential distributions caused by electron irradiation, which are consistent with the experiment data. In Figures 3a-3c, the simulations show that the charges created by electron irradiation increased with the incident electron intensity, and the electric potentials at the surface of the microfibril were estimated to be $45.3,71.8$, and $105.9 \mathrm{~V}$, respectively.

To obtain more detailed information of the charging effect, we present reconstructed amplitude images of a microfibril in Figure 4 from an amplitude reconstruction process of an electron holography technique (Shindo et al., 2009). In Figure 4a, a sharp dark contrast line is evident along the right side surface, as indicated by a small arrow. The strong dark contrast becomes clearer and thicker around the right top region of the microfibril as the incident electron intensity increases. The change in contrast evident in Figures $4 a-4 c$ indicates a change in the electric potential distribution around the top of the microfibril of the sciatic nerve tissue. The cause of the strong dark contrast around nonconductive specimens, such as microfibrils of the sciatic nerve tissue, should be considered taking into account the movements of charges, i.e., the dynamic motions of the secondary electrons and the drift of charged specimens. Detailed discussion will be given elsewhere.

\section{CONCLUSIONS}

The charging effects of a microfibril of sciatic nerve tissues were investigated using bright-field imaging and electron holography. The results are summarized as follows.

1. A strong charging effect was confirmed at the top of a linear microfibril by bright-field imaging.

2. The electric potential distribution around a charged microfibril was visualized by the phase reconstruction pro- 

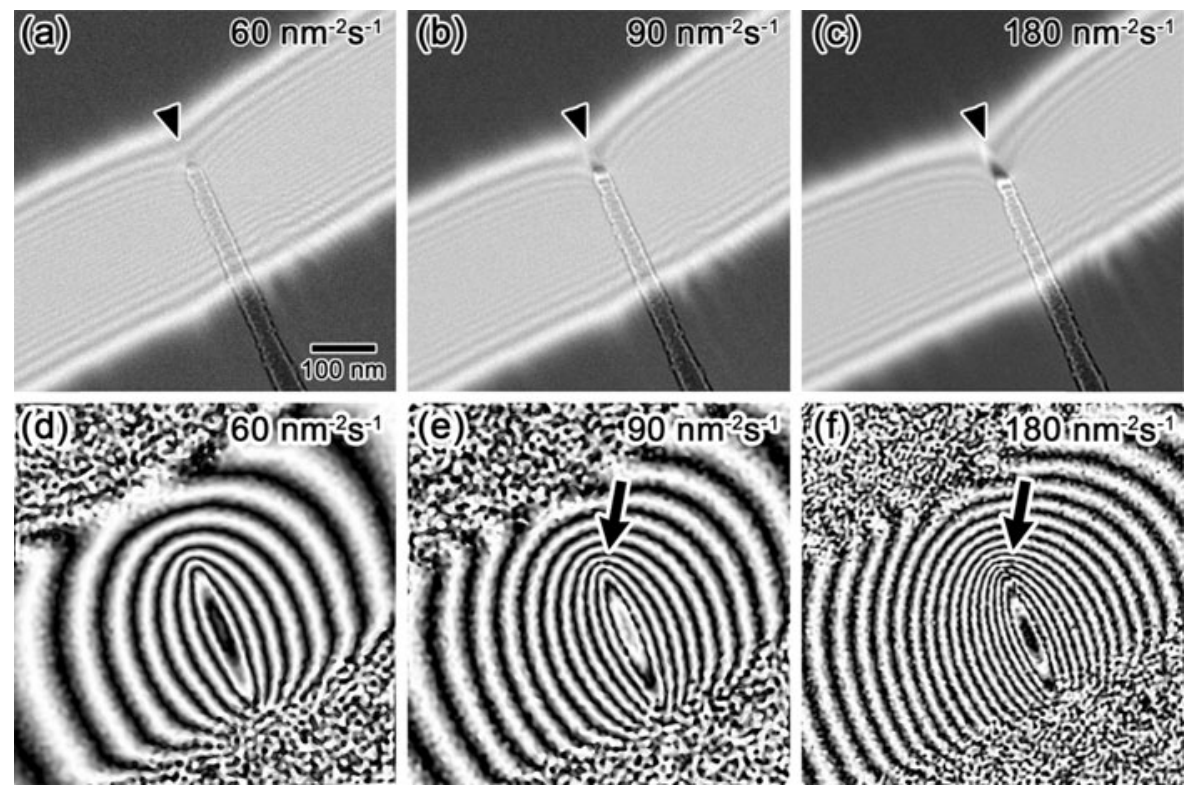

Figure 2. Electron holography results showing the electric potential distribution around a charged microfibril with various incident electron densities: $(\mathbf{a}-\mathbf{c})$ electron holograms and $(\mathbf{d}-\mathbf{f})$ the corresponding reconstructed phase images.

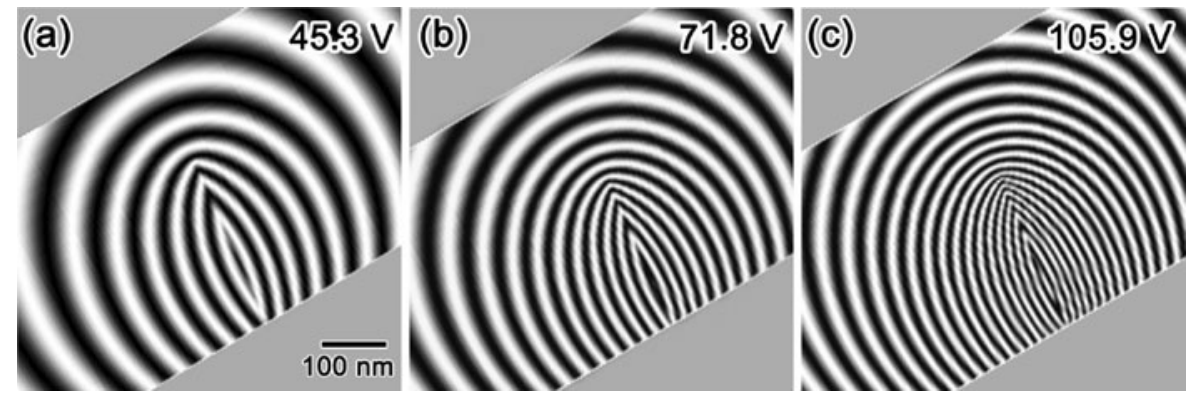

Figure 3. Simulation results showing electric potential distributions. The simulations corresponding to (a-c) were performed based on the assumption that the electric potentials at the surface of the microfibril were $45.3,71.8$, and $105.9 \mathrm{~V}$, respectively.
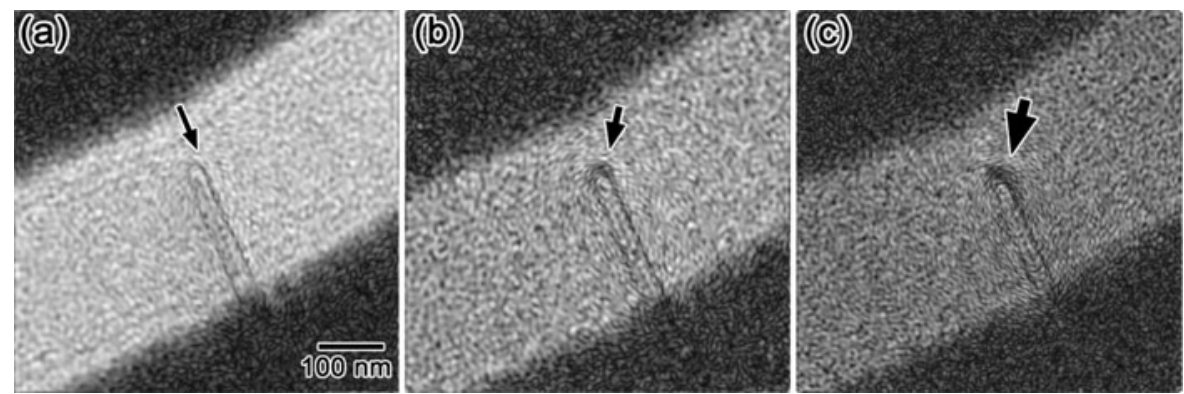

Figure 4. a-c: Reconstructed amplitude images of a microfibril of sciatic nerve tissues with change in the incident electron density. These images correspond to the reconstructed phase images shown in Figure 2.

cess of electron holography. The electric potential around the microfibril was quantitatively evaluated by simulations that take into account the reference wave modulation due to the long-range electric field.

3. The reconstructed amplitude images revealed the fluctuation of electric potential distribution around the microfibril.

\section{ACKNOWLEDGMENTS}

The authors thank Dr. Yasukazu Murakami for useful discussions. This work was supported by the Global COE Program "Materials Integration (International Center of Education and Research), Tohoku University," the Ministry of Education, Culture, Sports, Science and Technology (MEXT) of 
Japan and by a Grant-in-Aid for Scientific Research (S) (No. 19106002) from the Japan Society for the Promotion of Science (JSPS).

\section{REFERENCES}

JACKsON, J.D. (1998). Classical Electrodynamics, 3rd ed. New York, Chichester: John Wiley \& Sons.

Kotera, M., Yamaguchi, K. \& Suga, H. (1999). Dynamic simulation of electron-beam-induced charging up of insulators. Jpn J Appl Phys 38(12B), 7176-7179.

LI, D.J. \& Gu, H.Q. (2002). Cell attachment on diamond-like carbon coating. Bull Mater Sci 25(1), 7-13.

Matteucci, G., Missiroli, G. \& Pozzi, G. (1997). Simulations of electron holograms of long range electrostatic field. Scanning Microsc 11, 367-374.

Matteucci, G., Missiroli, G.F., Muccini, M. \& Pozzi, G. (1992). Electron holography in the study of the electrostatic fieldsThe case of charged microtips. Ultramicroscopy 45(1), 77-83.

Meyza, X., Goeuriot, D., Guerret-Piecourt, C., Treheux, D. \& Fitting, H.J. (2003). Secondary electron emission and selfconsistent charge transport and storage in bulk insulators: Application to alumina. J Appl Phys 94(8), 5384-5392.

MunGer, B.L. (1977). The problem of specimen conductivity in electron microscopy. In Scanning Electron Microscopy I, Johari, O. (Ed.), pp. 481-490. Chicago: IITRI.

Ohno, S., Hora, K., Furukawa, T. \& Oguchi, H. (1992). Ultrastructural-study of the glomerular slit diaphragm in fresh unfixed kidneys by a quick-freezing method. Virchows Arch B Cell Pathol Incl Mol Pathol 61(5), 351-358.

Okada, H., Shindo, D., Kim, J.J., Murakami, Y. \& Kawase, H. (2007). Triboelectricity evaluation of single toner particle by electron holography. J Appl Phys 102, 054908/1-5.
Rodnyansky, A., Warburton, Y.J. \& Hanke, L.D. (2000). Segregation in hot-dipped galvanized steel. Surf Interface Anal 29(3), 215-220.

Shi, X., Briseno, A.L., Sanedrin, R.J. \& Zhou, F. (2003). Formation of uniform polyaniline thin shells and hollow capsules using polyelectrolyte-coated microspheres as templates. Macromolecules 36, 4093-4098.

Shindo, D., Kim, J.J., Kim, K.H., Xia, W.X., Ohno, N., FujiI, Y., Terada, N. \& Ohno, S. (2009). Determination of orbital location of electron-induced secondary electrons by electric field visualization. J Phys Soc Jpn 78(10), 104802/1-8.

Shindo, D., Kim, J.J., Xıa, W.X., Kim, K.H., Ohno, N., Fujiı, Y., Terada, N. \& Ohno, S. (2007). Electron holography on dynamic motion of secondary electrons around sciatic nerve tissues. J Electron Microsc 56(1), 1-5.

Shindo, D. \& Oikawa, T. (2002). Analytical Electron Microscopy for Materials Science. Tokyo: Springer-Verlag.

Shindo, D., Park, Y.G., Murakami, Y., Gao, Y., Kanekiyo, H. \& Hirosawa, S. (2003). Electron holography of Nd-Fe-B nanocomposite magnets. Scr Mater 48(7), 851-856.

Touzin, M., Goeuriot, D., Guerret-Piecourt, C., Juve, D., Treheux, D. \& FitTing, H.J. (2006). Electron beam charging of insulators: A self-consistent flight-drift model. J Appl Phys 99(11), 114110/1-14.

Wepf, R., Amrein, M., Burkli, U. \& Gross, H. (1991). Platinum iridium carbon-a high-resolution shadowing material for TEM, STM and SEM of biological macromolecular structures. $J$ Microsc 163, 51-64. 